\title{
An alternative proof of Nyblom's results and a generalisation
}

\author{
A. David Christopher \\ Department of Mathematics, The American College \\ Tamil Nadu, India \\ e-mail: davchrame@yahoo.co.in
}

Received: 2 April 2019

Revised: 27 June 2020

Accepted: 30 June 2020

Abstract: Let $n$ be a positive integer and $k$ be a non-negative integer. We define

$$
p(n, k)=\left\{\begin{array}{lll}
n(n+k), & \text { if } k \equiv 0 \quad(\bmod 2) ; \\
\frac{n(n+k)}{2}, & \text { if } k \equiv 1 \quad(\bmod 2),
\end{array}\right.
$$

and $D(n, k)$ to be the number of ways $n$ can be expressed as a difference of two elements from the sequence $p(n, k)$. Nyblom found closed expressions for $D(n, 0)$ and $D(n, 1)$ in terms of some restricted number-of-divisors functions. Here we re-establish these two results of Nyblom in a relatively simple way. Along with the other interpretations for $D(n, k)$, an expression for $D(n, k)$ is presented in terms of restricted form of $D(n, 0)$ and $D(n, 1)$. Also we consider another function due to Nyblom, denoted $p_{D}(n)$, which counts the number of partitions of $n$ with parts in arithmetic progression having common difference $D$. Nyblom and Evan found a simple expression for $p_{2}(n)$ and put $p_{D}(n)$ in terms of a divisor-counting functions when $D \geq 3$. Here we re-establish Nyblom's expression for $p_{2}(n)$, and find equinumerous expressions for $p_{D}(n)$ when $D \geq 3$. Finally, we present the following generalised version of $D(n, k)$ : given a set of positive integers say, $A$, we denote by $D(n, A)$, the number of ways $n$ can be written as a difference of two elements from the set $A$. And we express $D(n, A)$ in terms of partition enumerations when some restrictions are imposed upon the elements of $A$. We close with the hint that, boundedness of $D(n, A)$ together with the divergence of $\sum_{a \in A} \frac{1}{a}$ disproves Erdôs arithmetic progression conjecture.

Keywords: Square numbers, Triangular numbers, Representation as difference of integers. 2010 Mathematics Subject Classification: Primary 11A67, Secondary 11B34. 


\section{Introduction}

Nyblom [6] converted the problem of finding the number of representation of integers as a difference of two squares to a system of linear Diophantine equation and thereby got the following expression:

$$
S(n)=\frac{1}{2}\left(\tau_{e}(n)+(-1)^{n+1} \tau_{o}(n)+\frac{1+(-1)^{\tau(n)+1}}{2}\right)
$$

where $S(n)$ denote the number of ways $n$ can be expressed as a difference of two square numbers, $\tau_{e}(n)\left(\right.$ resp. $\left.\tau_{o}(n)\right)$ denote the number of even (resp. odd) positive divisors of $n$ and $\tau(n)$ denote the number of positive divisors of $n$. In Section 2, we establish this expression in a simple and different way by counting a particular kind of divisors of $n$; this counting has already been done in a paper by the author [2].

In one of the other paper of Nyblom [5] following expression was derived:

$$
T(n)=\tau_{o}(n),
$$

where $T(n)$ denote the number of ways $n$ can be expressed as a difference of two triangular numbers. Even to derive this expression, Nyblom employed a technique similar to the one used in deriving (1). In Section 2, we establish (2) by counting another kind of divisors of $n$; essentials of this counting has been derived already in the same paper of the author [2].

Now, we define a sequence which is a common generalisation of square number sequence and triangular number sequence and find the expression of the above kind when elements of this sequence come into play.

Definition 1.1. Let $n$ be a positive integer and $k$ be a non-negative integer. We define

$$
p(n, k)=\left\{\begin{array}{lll}
n(n+k), & \text { if } k \equiv 0 \quad(\bmod 2) \\
\frac{n(n+k)}{2}, & \text { if } k \equiv 1 \quad(\bmod 2)
\end{array}\right.
$$

and $D(n, k)$ to be the number of ways $n$ can be expressed as a difference of two elements from the sequence $p(n, k)$.

From this definition, it follows that:

$$
S(n)= \begin{cases}D(n, 0)-1, & \text { if } \delta(n)=1 \\ D(n, 0), & \text { if } \delta(n)=0,\end{cases}
$$

where $\delta$ denotes the characteristic function of square numbers. Moreover, we have

$$
T(n)=D(n, 1) .
$$

The sequences $p(n, k)$ for the initial cases $k=2,3,4,5,6$ (with comments) can be found, respectively, in A005563, A000096, A028347, A055998 and A028560 of Online Encyclopedia of Integer Sequences. Following result concerning $p(n, k)$ is straightforward. 
Lemma 1.2. Let $n$ be a positive integer and let $k$ be a non-negative integer. Then

$$
p(n, k)= \begin{cases}p(n+1, k-2)-(k-1), & \text { if } k \equiv 0 \quad(\bmod 2) ; \\ p(n+1, k-2)-\frac{k-1}{2}, & \text { if } k \equiv 1 \quad(\bmod 2) .\end{cases}
$$

This lemma is instrumental in deriving expressions (which are presented in Section 2) for $D(n, k)$ in a restricted form of $D(n, 0)$ and $D(n, 1)$.

Next we are concerned with another result of Nyblom and Evan. The following definition is essential for mentioning that result.

Definition 1.3. Let $n$ and $D$ be two positive integers. Then the function $p_{D}(n)$ is defined to be the number of partitions of $n$ with parts in arithmetic progression having common difference $D$.

Note that, in the above definition, we consider the partition $(n)$ of $n$ as a partition in an arithmetic progression.

R. Cook and D. Sharp [1] found a necessary and sufficient condition for an integer $n$ to be written as a sum of arithmetic progression. In this sequence, Nyblom and Evans [7] defined the function $p_{D}(n)$ and obtained that:

$$
p_{2}(n)=\frac{1}{2}\left(\tau(n)-2+\frac{(-1)^{\tau(n)+1}+1}{2}\right)+1,
$$

and also they have obtained an interpretation for the term $p_{D}(n)$ in terms of a divisor-counting function. A. O. Munagi [4] pointed out that such an expression for $p_{D}(n)$ by Nyblom and Evans is a complicated one. In Section 2 we re-establish (6) and also we find alternative interpretation for $p_{D}(n)$ which is relatively simple to that of Nyblom and Evans.

Section 3 is concerned with a generalisation of the above problem, that is, we are concerned with the number of ways a positive integer $n$ can be expressed as a difference of two elements of a given set of positive integers. Following definition forms a basis for Section 3.

Definition 1.4. Let $n$ be a positive integer and let $A$ be a set of positive integers. Then we define the function $D(n, A)$ to be the number of ways $n$ can be expressed as a difference of two elements from the the set $A$.

In Section 3 we found that the function $D(n, A)$ is equinumerous with a kind of partitions involving the set $A$ with some additional constraints. We close by mentioning a connection between boundedness of $D(n, A)$ and Erdôs arithmetic progression conjecture.

Notations and definitions in this section bears the same meaning throughout the article when used.

\section{Proof and an Extension of Nyblom's results}

\subsection{Alernative proof of Nyblom's results}

In this section we give an alternative and simple proofs of Nyblom's expressions [5, 6].

Let $n$ be a positive integer. Suppose that $n$ can be written as a difference of two squares; both non-zero. Then we have the following equalities: 


$$
\begin{aligned}
n & =y^{2}-x^{2} \\
& =(x+d)^{2}-x^{2} \\
& =2 d x+d^{2} .
\end{aligned}
$$

This gives the following congruence:

$$
\frac{n}{d}-d \equiv 0 \quad(\bmod 2)
$$

with $\frac{n}{d}-d \geq 1$.

Conversely, every solution to the congruence above gives a representation of $n$ as a difference of two squares; both non-zero.

In [2] to conclude Theorem 5, the number of divisors $d$ of $n$ satisfying the congruence (7) was counted. Now in view of the above observations, we equate that counting with $D(n, 0)$.

$$
D(n, 0)=\left\{\begin{array}{lll}
\frac{\tau(n)}{2} & \text { if } n \equiv 1 \quad(\bmod 2) \text { and } \delta(n)=1 \\
\frac{\tau(n)-1}{2} & \text { if } n \equiv 1 \quad(\bmod 2) \text { and } \delta(n)=0 \\
\frac{(\beta-1) \tau\left(\frac{n}{2^{\beta}}\right)-1}{2} & \text { if } n \equiv 0 \quad(\bmod 2) \text { and } \delta(n)=1 \\
\frac{(\beta-1) \tau\left(\frac{n}{2^{\beta}}\right)}{2} & \text { if } n \equiv 0 \quad(\bmod 2) \text { and } \delta(n)=0
\end{array}\right.
$$

where $\beta$ is the highest power of 2 that divides $n$.

Now we observe that (8) is a disguised form of (1).

Case i. When $n \equiv 1(\bmod 2)$ and $\delta(n)=0$, in accordance with $(1)$, we can write

$$
\begin{aligned}
D(n, 0) & =\frac{1}{2}\left(\tau_{e}(n)+\tau_{o}(n)+\frac{1+1}{2}\right)-1 \\
& =\frac{1}{2}(\tau(n)+1)-1 \\
& =\frac{\tau(n)-1}{2} .
\end{aligned}
$$

Case ii. When $n \equiv 1(\bmod 2)$ and $\delta(n)=1$, in accordance with (1), we can write

$$
\begin{aligned}
D(n, 0) & =\frac{1}{2}\left(\tau_{e}(n)+\tau_{o}(n)+\frac{1-1}{2}\right) \\
& =\frac{1}{2} \tau(n) .
\end{aligned}
$$

Case iii. When $n \equiv 0(\bmod 2)$ and $\delta(n)=1$, by $(1)$, we have

$$
\begin{aligned}
D(n, 0) & =\frac{1}{2}\left(\tau_{e}(n)-\tau_{o}(n)+1\right)-1 \\
& =\frac{1}{2}\left(\beta \tau\left(\frac{n}{2^{\beta}}\right)-\tau\left(\frac{n}{2^{\beta}}\right)+1\right)-1 \\
& =\frac{1}{2}\left((\beta-1) \tau\left(\frac{n}{2^{\beta}}\right)-1\right) .
\end{aligned}
$$


Case iv. When $n \equiv 0(\bmod 2)$ and $\delta(n)=0$, as before, we have

$$
\begin{aligned}
D(n, 0) & =\frac{1}{2}\left(\tau_{e}(n)-\tau_{o}(n)\right) \\
& =\frac{1}{2}\left((\beta-1) \tau\left(\frac{n}{2^{\beta}}\right)\right) .
\end{aligned}
$$

Thus we have obtained a proof of (1).

Now we turn to the derivation of (2). Suppose that $n$ can be written as a difference of two triangular numbers. Then we have

$$
\begin{aligned}
n & =\frac{y(y+1)}{2}-\frac{x(x+1)}{2} \\
& =\frac{(x+d)(x+d+1)}{2}-\frac{x(x+1)}{2} .
\end{aligned}
$$

This gives

$$
\frac{2 n}{d}-d=2 x+1
$$

for some non-negative integer $x$.

From this, we observe that $T(n)$ equals the number of divisors $d$ of $2 n$ which satisfies the following:

1. $d<\sqrt{2 n}$;

2. Either $d$ is odd or $d=2^{\beta+1} k$ for some integer $k$, where $\beta$ as before denotes the highest power of 2 that divides $n$.

Now again from the lines of proof of Theorem 5 in [2] we can write

$$
T(n)= \begin{cases}\frac{\tau(2 n)}{2}-\frac{(\beta+1-1) \tau\left(\frac{2 n}{2^{\beta+1}}\right)}{2} & \text { if } \delta(2 n)=0 \\ \frac{\tau(2 n)-1}{2}-\frac{(\beta+1-1) \tau\left(\frac{2 n}{2^{\beta+1}}\right)-1}{2} & \text { if } \delta(2 n)=1 .\end{cases}
$$

Above equality is equivalent to

$$
T(n)= \begin{cases}\frac{\tau_{e}(2 n)+\tau_{o}(2 n)-\left(\tau_{e}(2 n)-\tau_{o}(2 n)\right)}{2} & \text { if } \delta(2 n)=0 \\ \frac{\tau_{e}(2 n)+\tau_{o}(2 n)-1}{2}-\frac{\tau_{e}(2 n)-\tau_{o}(2 n)-1}{2} & \text { if } \delta(2 n)=1 .\end{cases}
$$

Consequently, we get

$$
\begin{aligned}
T(n) & =\tau_{o}(2 n) \\
& =\tau_{o}(n) .
\end{aligned}
$$

Thus Nyblom's expression for $T(n)$ is re-established.

Now we turn to another result of Nyblom and Evans about $p_{2}(n)$ mentioned in (6). Our derivation is based upon the following observation: if $n$ can be written as a sum of the terms in arithmetic progression with common difference $D$, then we have: 


$$
\begin{aligned}
n & =a+(a+D)+\cdots+(a+(m-1) D) \\
& =m a+\frac{m(m-1)}{2} D .
\end{aligned}
$$

This gives

$$
2 n=2 m a+m^{2} D-m D .
$$

When $D=2$, we have

$$
\frac{n}{m}-m=a-1 \text {. }
$$

Thus $p_{2}(n)$ equals the number of divisors of $n$ satisfying the above equation. Since $a$ is positive, $p_{2}(n)$ equals the number of divisors $m$ of $n$ satisfying the inequality $m \leq \sqrt{n}$. One can see that if $n$ is a non-square then the mapping $m \rightarrow \frac{n}{m}$ is one-one and non-fixed. If $n$ is a square number then the mapping $m \rightarrow \frac{n}{m}$ is again one-one and non-fixed when $m<\sqrt{n}$ and $m$ fixes to itself under the map $m \rightarrow \frac{n}{m}$ if $m=\sqrt{n}$. These observations lead to the conclusion that

$$
p_{2}(n)= \begin{cases}\frac{\tau(n)}{2} & \text { if } \delta(n)=0 \\ \frac{\tau(n)-1}{2}+1 & \text { if } \delta(n)=1\end{cases}
$$

Thus the expression for $p_{2}(n)$ due to Nyblom and Evans [7] follows.

Next we give an alternative interpretation for $p_{2 D}(n)$ and $p_{2 D+1}(n)$ in terms of a divisorcounting function which is the contention of the following result.

Theorem 2.1. Let $n$ and $D$ be positive integers. Then we have

1. $p_{2 D}(n)$ equals the number of divisors $m$ of $n$ such that $m<\frac{D+\sqrt{D^{2}+4 D n}}{2 D}$.

2. $p_{2 D-1}(n)$ equals the number of divisors $m$ of $2 n$ such that $m<\frac{\left(D-\frac{1}{2}\right)+\sqrt{\left(D-\frac{1}{2}\right)^{2}+2(2 D-1) n}}{2 D-1}$.

Proof. If common difference is $2 D$ then from (9) it follows that

$$
\frac{n}{m}-(m-1) D=a>0
$$

This can be put as a quadratic inequality

$$
m^{2} D-m D-n<0 .
$$

After factoring we get

$$
\left(m-\frac{D-\sqrt{D^{2}+4 D n}}{2 D}\right)\left(m-\frac{D+\sqrt{D^{2}+4 D n}}{2 D}\right)<0 .
$$

Since the first factor is always positive we have

$$
\left(m-\frac{D+\sqrt{D^{2}+4 D n}}{2 D}\right)<0 .
$$


Also from (9) we conclude that $m$ must be a divisor of $n$. Moreover every divisor $m$ of $n$ satisfying the above inequality contribute to the equality (11). Hence first part of the result follows. Similar approach will settle the second part.

Definition 2.2. Let $n$ be a positive integer. By unrestricted partition of $n$, we mean a nonincreasing sequence of integers say $\left(a_{1}, a_{2}, \ldots, a_{k}\right)$ such that $a_{1}+a_{2}+\cdots+a_{k}=n$. We use $p_{D}^{u}(n)$ to denote the number of unrestricted partitions of $n$ whose parts follows arithmetic progression with common difference $D$.

Theorem 2.3. Let $n$ be a positive integer. Then we have

$$
p_{D}^{u}(n)=\left\{\begin{array}{lll}
\tau(n) & \text { if } D \equiv 0 & (\bmod 2) ; \\
2 \tau_{o}(n) & \text { if } D \equiv 1 & (\bmod 2) .
\end{array}\right.
$$

Proof. Assume that the common difference is $2 D$, an even integer. Then from (9) it follows that

$$
\frac{n}{m}-(m-1) D=a \text {. }
$$

Since $a$ can be any integer, each divisor $m$ of $n$ contribute an $a$. Consequently, corresponding to each divisor $m$ of $n$ there exists an unrestricted partition of the said type and vice versa. Hence the first part of the result follows.

Assume that the common difference is $2 D+1$, an odd integer. Then from (9) it follows that

$$
\frac{2 n}{m}-(2 D+1)(m-1)=2 a .
$$

Choosing divisors $m$ of $2 n$ such that either $m$ is odd or $m$ is of the form $m=2^{\beta}(2 r+1)$, where $\beta$ is the highest power of 2 that divides $2 n$ will alone contribute an even integer $2 a$ at the right side of (14). As one can see, the number of such divisors is $\tau_{o}(2 n)+\tau_{o}(2 n)$, which is equal to $2 \tau_{o}(n)$. Now the result follows.

Remark 2.4. It is interesting to note that the function $p_{D}(n)$ is independent of the value of $D$, whereas it is dependent on the parity of $D$ and the factorisation of $n$.

\subsection{Other interpretations of the term $D(n, k)$}

Now we confine to the expression $D(n, k)$ when $k$ exceeds 1 .

Case i. Assume that $k \equiv 1(\bmod 2)$. If $n$ can be written as a difference of two members from the sequence $p(n, k)$, then we have

$$
\begin{aligned}
n & =p(y, k)-p(x, k) \\
& =p(x+d, k)-p(x, k) \\
& =\frac{(x+d)(x+d+k)}{2}-\frac{x(x+k)}{2} .
\end{aligned}
$$

This implies that

$$
\frac{2 n}{d}-d=2 x+k .
$$

From this observation, we have the following result. 
Theorem 2.5. Let $k \equiv 1(\bmod 2)$ be a positive integer and let $n$ be a positive integer. Then $D(n, k)$ counts the divisors $d$ of $2 n$ such that $\frac{2 n}{d}-d$ is an odd integer not less than $k$.

Corollary 2.6. Let $k \equiv 1(\bmod 2)$ be a positive integer and let $n$ be a positive integer such that: for each divisor $d$ of $2 n, \frac{2 n}{d}-d$ is an odd integer not less than $k$. Then $D(n, k)=D(n, 1)$.

Case ii. Assume that $k \equiv 0(\bmod 2)$. If $n$ can be written as a difference of two members from the sequence $p(n, k)$, then we have

$$
\begin{aligned}
n & =p(y, k)-p(x, k) \\
& =p(x+d, k)-p(x, k) \\
& =(x+d)(x+d+k)-x(x+k) .
\end{aligned}
$$

This implies that

$$
\frac{n}{d}-d=2 x+k
$$

From this observation, we have the following result.

Theorem 2.7. Let $k \equiv 0(\bmod 2)$ be a positive integer and let $n$ be a positive integer. Then $D(n, k)$ counts the divisors $d$ of $n$ such that $\frac{n}{d}-d$ is an even integer not less than $k$.

Corollary 2.8. Let $k \equiv 0(\bmod 2)$ be a positive integer and let $n$ be a positive integer such that: for each divisor $d$ of $n, \frac{n}{d}-d$ is an even integer not less than $k$. Then $D(n, k)=D(n, 0)$.

To mention some of the forthcoming results, some basic terminologies from partition theory are required.

Definition 2.9. Let $n$ be a positive integer. By a partition of $n$, we mean a sequence of nonincreasing positive integers say $\pi=\left(a_{1}, a_{2}, \ldots, a_{k}\right)$ such that $a_{1}+a_{2}+\cdots+a_{k}=n$. Each $a_{i}$ is called a part of $\pi$ and the number of times part $a_{i}$ occurs is referred as the frequency of $a_{i}$. Each element in the set of parts of $\pi$ is called a size of $\pi$.

We observe that (15) can be put in the form: $2 n=\left(f_{1}+f_{2}\right) f_{1}+f_{1}\left(f_{2}+k\right)$ for some positive integers $f_{1}$ and $f_{2}$. This leads to the following result.

Theorem 2.10. Let $n$ be a positive integer and let $k \equiv 1(\bmod 2)$ be a positive integer. Then $D(n, k)$ equals the number of partitions of $2 n$ with sizes $f_{1}+f_{2}$ and $f_{1}$ with their respective frequencies $f_{1}$ and $f_{2}+k$.

Similar observation on (16) gives the following result.

Theorem 2.11. Let $n$ be a positive integer and let $k \equiv 0(\bmod 2)$ be a positive integer. Then $D(n, k)$ equals the number of partitions of $n$ with sizes $f_{1}+f_{2}$ and $f_{1}$ with their respective frequencies $f_{1}$ and $f_{2}+k$.

We observe that: $p(m, 2 k-1)=k+(k+1)+\cdots+(k+m-1)$. Therefore, if $n$ can be written as a difference of two elements from $p(m, 2 k-1)$, then this difference gives a partition of $n$ with consecutive integers as parts with least part not less than $k$ and vice versa. This gives the following result. 
Theorem 2.12. Let $n$ be a positive integer. Then $D(n, 2 k-1)$ equals the number of partitions of $n$ with consecutive integers as parts with least part not less than $k$.

Since $p(m, 2 k)=(2 k+1)+(2 k+3)+\cdots+(2 k+2 m-1)$, as in the previous case, we have the following result.

Theorem 2.13. Let $n$ be a positive integer. Then $D(n, 2 k)$ equals the number of partitions of $n$ with consecutive odd integers as parts with least part not less than $2 k+1$.

Remark 2.14. From the above results we see that the following enumerations are equivalent and are equal with $D(n, k)$ when $k$ is an odd integer:

1. Number of divisors $d$ of $2 n$ such that $\frac{2 n}{d}-d$ is an odd integer not less than $k$.

2. Number of partitions of $2 n$ with sizes $f_{1}+f_{2}$ and $f_{1}$ with their respective frequencies $f_{1}$ and $f_{2}+k$.

3. Number of partitions of $n$ with consecutive integers as parts with least part not less than $\frac{k+1}{2}$.

Similarly following enumerations are equivalent and are equal with $D(n, k)$ when $k$ is even:

1. Number of divisors $d$ of $n$ such that $\frac{n}{d}-d$ is an even integer not less than $k$.

2. Number of partitions of $n$ with sizes $f_{1}+f_{2}$ and $f_{1}$ with their respective frequencies $f_{1}$ and $f_{2}+k$.

3. Number of partitions of $n$ with consecutive odd integers as parts with least part not less than $k+1$.

To realise the essence of Lemma 1.2 we need the following definition.

Definition 2.15. Let $n$ and $r$ be two positive integers and $k$ be a non-negative integer. Denote by $D(n, k, r)$, the number of ways $n$ can be expressed as a difference of two elements from the sequence $\{p(n, k)\}_{n \geq r+1}$.

As a consequence of Lemma 1.2, the function $D(n, k)$ can be written in terms of $D(n, k, r)$ which is the contention of the following result.

Theorem 2.16. Let $n$ be a positive integer and $k$ be a non-negative integer. Then we have

$$
D(n, k)=\left\{\begin{array}{lll}
D\left(n, 1, \frac{k-1}{2}\right) & \text { if } k \equiv 1 & (\bmod 2) ; \\
D\left(n, 0, \frac{k}{2}\right) & \text { if } k \equiv 0 & (\bmod 2) .
\end{array}\right.
$$

Proof. Assume $k \equiv 1(\bmod 2)$. In view of Lemma 1.2 we can write

$$
\begin{aligned}
D(n, k) & =D(n, k-2,1) \\
& =D(n, k-4,2)
\end{aligned}
$$

and so on. Then after the $\frac{k-1}{2}$ times of repeated application of Lemma 1.2 as above we get

$$
D(n, k)=D\left(n, 1, \frac{k-1}{2}\right),
$$

which is the expected end. Similar application of Lemma 1.2 serves good in odd case. 


\section{Generalised version of Nyblom's results}

\subsection{An interpretation for $D(n, A)$}

Recall from Definition 1.4 that $D(n, A)$ is the number of ways $n$ can be written as a difference of two elements from the set $A$. We presumably take $\operatorname{gcd}(A)=1$. For otherwise, integer $n$ which are non-multiples of $\operatorname{gcd}(A)$ cannot be expressed as a difference of elements from $A$. Following theorem gives an interpretation for $D(n, A)$ in terms of an integer partition enumeration.

Theorem 3.1. Let $n$ be a positive integer and let $P=\left\{a_{1}, a_{2}, \ldots\right\}$ be a set of positive integers with $\operatorname{gcd}(P)=1$ and $a_{1}<a_{2}<\cdots$. Define $s_{n}=a_{1}+a_{2}+\cdots+a_{n}$ for every $n \geq 1$ and $A=\left\{s_{1}, s_{2}, \ldots\right\}$. Then $D(n, A)$ equals the number of partitions of $n$ with parts as consecutive elements of $P$.

Proof. If $n$ can be written as difference of two elements from $A$ then we have

$$
\begin{aligned}
n & =s_{k}-s_{r} \\
& =a_{r+1}+a_{r+2}+\cdots+a_{k}
\end{aligned}
$$

for some $k>r$. Thus this difference gives a representation of $n$ as a sum of consecutive members of $P$.

On the other side, if

$$
n=a_{r+1}+a_{r+2}+\cdots+a_{k}
$$

with $k>r$, then we can write

$$
n=s_{k}-s_{r} .
$$

Hence, the representation as a sum of consecutive elements of $P$ gives a representation of $n$ as a difference of elements of $A$. This correspondence establishes the result.

Corollary 3.2. Let $n$ be a positive integer. Then we have

1. The number of ways $n$ can be written as a difference of squares of triangular numbers equals the number of ways $n$ can be written as the sum of consecutive cubes.

2. No prime can be expressed as a sum of consecutive cubes.

3. The number of ways $n$ can be expressed as a difference of two Fibonacci numbers equals the number partitions of $n$ with parts as consecutive Fibonacci numbers.

Proof. Statements 1. and 2. follows from the identity

$$
1^{3}+2^{3}+\cdots+n^{3}=\left(\frac{n(n+1)}{2}\right)^{2} .
$$

Statement 3. follows from the identity:

$$
F_{0}+F_{1}+\cdots+F_{n}=F_{n+2}-1
$$




\subsection{Boundedness of $D(n, A)$ and Erdôs Arithmetic Progression Conjecture}

Erdős conjectured that, if $\sum_{a \in A} \frac{1}{a}$ diverges then $A$ contains arithmetic progression of arbitrary length. Now if we assume that the conjecture is true then the boundedness of $D(n, A)$ implies the convergence of $\sum_{a \in A} \frac{1}{a}$. For if $\sum_{a \in A} \frac{1}{a}$ is diverging, then since we assume the truthness of Erdős conjecture, for any given positive integer $k$ we have

$$
\{a, a+d, a+2 d, \cdots, a+k d\} \subset A
$$

for some positive integers $a$ and $d$. Consequently, $D(d, A) \geq k$. That is for any given positive integer $k$ we can find a positive integer $d$ such that $D(d, A) \geq k$. Thus $D(n, A)$ is unbounded.

Next we observe that the converse of the above statement need not be true; for we have the converging series $\sum_{n=1}^{\infty} \frac{1}{n^{2}}$ but $D\left(n,\left\{1^{2}, 2^{2}, \cdots\right\}\right)$ is not bounded. Based upon this discussion we state our closing result.

Theorem 3.3. If there exist a set of positive integer, say $A$, such that $\sum_{a \in A} \frac{1}{a}$ diverges and $D(n, A)$ is bounded, then the Erdós arithmetic progression conjecture fails.

Remark 3.4. If one finds a set of positive integers satisfying the hypothesis of the above theorem, then the Erdôs conjecture will be laid to rest.

\section{References}

[1] Cook, R., \& Sharp, D. (1995). Sums of arithmetic progressions, Fibonacci Quart., 33, $218-221$.

[2] Christopher, A. D. (2015). Partitions with Fixed Number of Sizes, J. Integer Seq., 18, Article 15.11.5.

[3] Mason, T. E. (1912). On the representation of an integer as the sum of consecutive integers, Amer. Math. Monthly, 19 (3), 46-50.

[4] Munagi, A. O. (2010). Combinatorics of integer partitions in arithmetic progression, Integers, 10, 73-82.

[5] Nyblom, M. A. (2001). On the Representation of the Integers as a Difference of non-consecutive Triangular numbers, Fibonacci Quart., 39 (3), 256-263.

[6] Nyblom, M. A. (2002). On the Representation of the Integers as a Difference of Squares, Fibonacci Quart., 40 (3), 243-246.

[7] Nyblom M. A., \& Evans, C. (2003). On the enumeration of partitions with summands in arithmetic progression, Australas. J. Combin., 28, 149-159. 